\title{
LITOTRICIA EXTRACORPÓREA EN LITIASIS EN PACIENTES CON DERIVACIÓN URINARIA
}

\author{
A. VEGA VEGA*, D. GARCÍA ALONSO*, L. PARRA MUNTANER**, \\ J.M. SÁNCHEZ MERINO**, J. GARCÍA ALONSO** \\ *Servicio de Urología. Obra Hospitalaria Nuestra Señora de Regla. León. \\ **Servicio de Urología del Hospital del Bierzo. Ponferrada. León.
}

Actas Urol Esp. 28 (5): 400-404, 2004

\section{RESUMEN}

LITOTRICIA EXTRACORPÓREA EN LITIASIS EN PACIENTES CON DERIVACIÓN URINARIA

Exponemos nuestra experiencia en el tratamiento de litiasis en pacientes con derivación urinaria. Hemos tratado 5 pacientes con litiasis en el tracto urinario superior tras cistectomía y derivación urinaria. El tratamiento se ha hecho con monoterapia por ondas de choque con el litotritor Lithostar Modularis de Siemens. El tamaño medio de las litiasis fue de 1,95 cm (rango 1 a 3,5 cm). El número medio de sesiones ha sido 2,6, con 3961 ondas de choque de media por sesión. Hemos conseguido un $100 \%$ de pacientes libres de litiasis, indicando que la litotricia extracorpórea tiene unos buenos resultados en el tratamiento de las litiasis en pacientes con derivación urinaria, siendo el tratamiento de elección en estos pacientes, con muy baja morbilidad y alta eficacia, comparable a los pacientes sin derivación urinaria.

PALABRAS CLAVE: Derivación urinaria. Litotricia. Litiasis.

\section{ABSTRACT \\ EXTRACORPOREAL SHOCK WAVE LITHOTRIPSY OF UROLITHIASIS IN PATIENTS WITH URINARY DIVERSION}

We evaluate the efficacy of ESWL in the management of calculi in patients with urinary diversion. We treated 5 patients who suffered from urinary lithiasis after urinary diversion post cistectomy. We managed the patients with extracorporeal shock wave lithotripsy in monotherapy with a Siemens Lithostar Modularis device. Mean stone size (long axis) was $1.95 \mathrm{~cm}$ (range 1 to $3.5 \mathrm{~cm}$ ). The mean shockwave number per session was 2.6 per patient. Stone free result was $100 \%$. We conclude that ESWL technique can provide acceptable results in patients with urinary diversion and can be used as first choice treatment in these type of patients due to its minimal morbidity and excellent results, equivalent to those achieved in patients without urinary diversion.

KEY WORDS: Urinary diversion. ESLW. Lithiasis.

$\mathrm{L}$ a litiasis urinaria en el tracto urinario superior es una complicación frecuente en las derivaciones urinarias ${ }^{1}$. Está favorecida por las alteraciones metabólicas, infecciosas y anatómicas. Las alteraciones metabólicas son debidas al uso de segmentos intestinales en la creación del conducto y de la neovejiga, así como a la resección intestinal ${ }^{2}$. Las alteraciones infecciosas que favorecen la litiasis son la colonización crónica o infecciones por bacterias ureasa positivas, favorecidas a su vez por el uso de catéteres ${ }^{3}$. Las alteraciones anatómicas que favorecen la formación 
de litiasis son por una parte la disminución del peristaltismo debido a la manipulación durante la intervención de los uréteres, así como la posibilidad de existencia de reflujo y/o estenosis en la unión de los uréteres a la neovejiga o al conducto urinario ileal ${ }^{4,5}$.

En el tratamiento de estas litiasis se ha empleado desde cirugía abierta en el pasado a cirugía percutánea y litotricia extracorpórea por ondas de choque en la actualidad ${ }^{6}$. Presentamos nuestra experiencia en el tratamiento de litiasis en el tracto urinario superior en pacientes con diversión urinaria mediante litotricia extracorpórea por ondas de choque.

\section{PACIENTES Y MÉTODOS}

Hemos tratado 5 pacientes en un periodo de 3 años entre enero del 2000 y diciembre del 2002, 2 mujeres y 3 varones con edades comprendidas entre los 47 y 77 años (edad media 61 años). En los 3 varones se hizo la cistectomía por cáncer vesical infiltrante, en una mujer por infiltración local de cáncer de colon y en la otra por extrofia vesical. La derivación urinaria fue en 3 casos a un conducto ileal tipo Bricker, en un caso vejiga ileal ortotópica y en otra derivación urinaria continente tipo Koch (Fig. 1).
Como antecedentes urológicos 2 pacientes presentan antecedentes de litiasis; 3 presentan infecciones de repetición y en otro el debut de la litiasis es por sepsis tras obstrucción por litiasis, el otro debuta como cólico; 3 pacientes tienen nefrostomía en el momento de ser tratados de la litiasis.

Hemos tratado 6 litiasis, un paciente con dos y el resto con una litiasis. La localización es en 4 casos izquierda y en uno derecha. En 3 casos la litiasis se sitúa en la pelvis renal y en otros 3 en el uréter. El tamaño de la litiasis oscila entre 1 y $3,5 \mathrm{~cm}$, con una media de $1,95 \mathrm{~cm}$ (Tabla I).

La litotricia se hizo de forma ambulatoria en todos los pacientes, hemos utilizado el litotritor Siemens Modularis, de enegia electromagnética. Todas las sesiones se han hecho con sedación con alfentanil y midazolam y se han usado antibióticos profilácticos tras las sesiones en todos ellos (Fig. 2).

\section{RESULTADOS}

Las complicaciones no han sido de importancia, con hematurias autorresolutivas, de baja intensidad tras la sesión de litotricia y sólo un caso de infección complicada que ha motivado su ingreso en el paciente con neovejiga ortotópica.
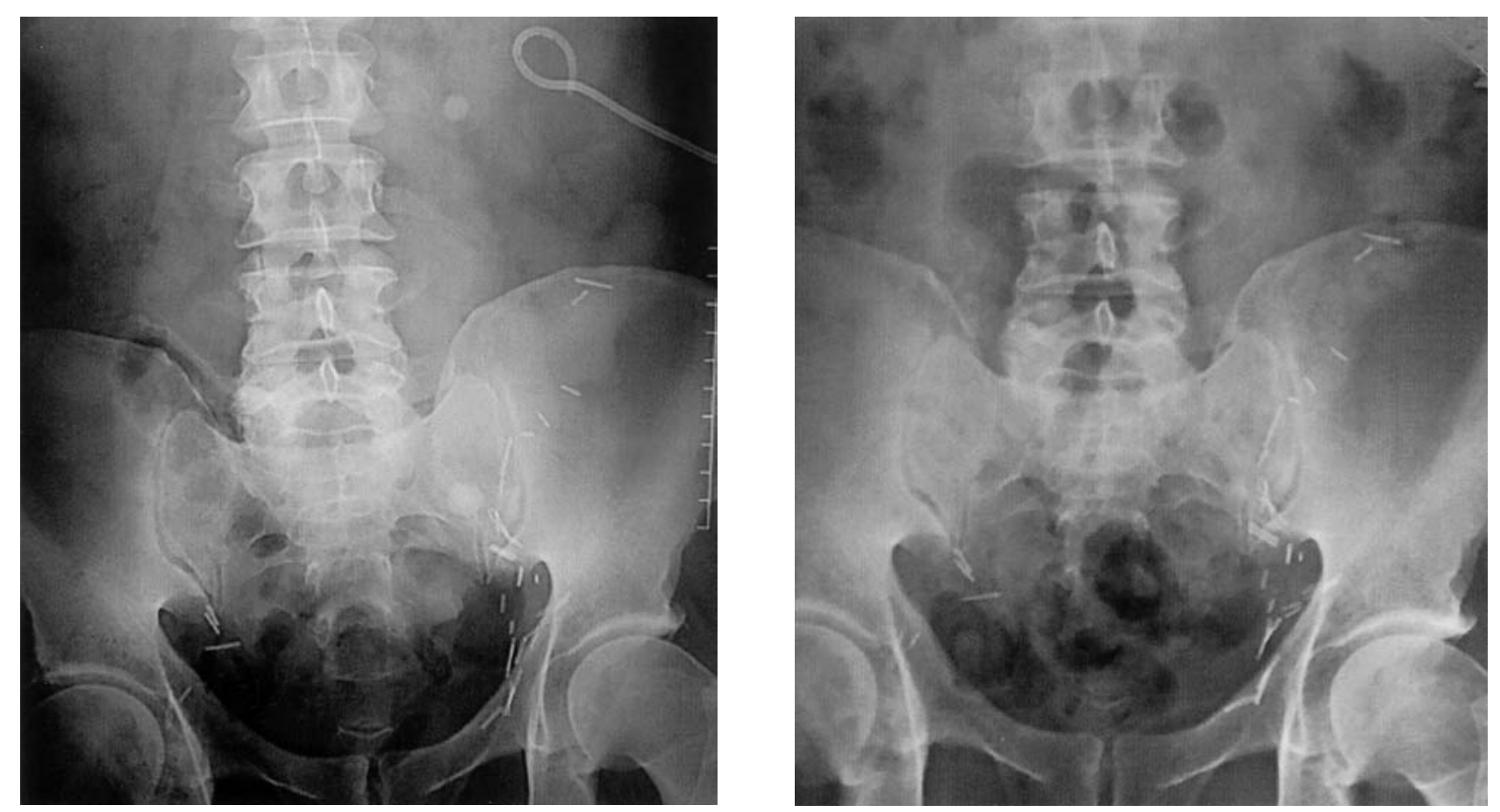

FIGURA 1. Paciente con neovejiga ileal ortotópica pre y postratamiento. 
TABLA I

CARACTERÍSTICAS DE LOS PACIENTES

\begin{tabular}{|c|c|c|c|c|c|c|c|}
\hline \multirow[b]{2}{*}{ Caso } & \multirow[b]{2}{*}{$\begin{array}{c}\text { Edad } \\
\text { (años) }\end{array}$} & \multirow[b]{2}{*}{ Sexo } & \multirow[b]{2}{*}{$\begin{array}{c}\text { Antecedentes } \\
\text { personales }^{2}\end{array}$} & \multicolumn{4}{|c|}{ Antecedente litiásico actual } \\
\hline & & & & $\begin{array}{c}\text { Localización } \\
\text { litiasis }\end{array}$ & Lado & Tamaño & Sintomatologia \\
\hline 1 & 56 & $\mathrm{v}$ & $\begin{array}{l}\text { Studer por neo } \\
\text { vesical grado III } \\
\text { pT3b NO MO } \\
\text { TVP en postq tto } \\
\text { con sintrom, con } \\
\text { HBPM flaxiparina } \\
0,6 \text { en tto con } \\
\text { litotricia }\end{array}$ & $\begin{array}{l}2 \text { litiasis } \\
\text { una en pelvis } \\
\text { renal y otra en } \\
\text { uréter (no } \\
\text { describo } \\
\text { localización por } \\
\text { estar alterada con } \\
\text { la intervención }\end{array}$ & Izquierdo & $\begin{array}{l}1,2 \mathrm{~cm} \\
\text { piélica y } \\
1,5 \mathrm{~cm} \text { la } \\
\text { ureteral }\end{array}$ & $\begin{array}{l}\text { Obstrucción } \\
\text { con infección, } \\
\text { se pone } \\
\text { nefrostomía } \\
\text { por sepsis }\end{array}$ \\
\hline 2 & 74 & $\mathrm{v}$ & $\begin{array}{l}\text { Bricker tras } \\
\text { cistoprostatectomía } \\
7 \text { años antes por } \\
\text { neo grado III }\end{array}$ & Pseudocoraliforme & Izquierdo & $3 \mathrm{~cm}$ & $\begin{array}{l}\text { Infección de } \\
\text { orina de } \\
\text { repetición }\end{array}$ \\
\hline 3 & 77 & $\mathrm{v}$ & $\begin{array}{l}\text { Bricker tras } \\
\text { cistoprostatectomía } \\
5 \text { años antes por } \\
\text { neo grado III }\end{array}$ & Uréter terminal & Izquierdo & $1 \mathrm{~cm}$ & Cólico \\
\hline 4 & 51 & $\mathrm{~m}$ & $\begin{array}{l}\text { Derivación } \\
\text { continente por } \\
\text { extrofia } 15 \text { años } \\
\text { antes, en la } \\
\text { actualidad con } \\
\text { nefrostomia } \\
\text { bilateral por } \\
\text { obstrucción } \\
\text { ureteral bilateral } \\
\text { por estenosis, } \\
\text { múltiples } \\
\text { intervenciones } \\
12 \text { litotricias } \\
\text { previas }\end{array}$ & Uréter terminal & Derecho & $1,5 \mathrm{~cm}$ & $\begin{array}{l}\text { Infección de } \\
\text { repetición por } \\
\text { nefrostomias }\end{array}$ \\
\hline 5 & 47 & $\mathrm{~m}$ & $\begin{array}{l}\text { Derivación no } \\
\text { continente tipo } \\
\text { bricker por } \\
\text { cistectomía por } \\
\text { infiltración por neo } \\
\text { de colon }\end{array}$ & Pelvis renal & Izquierdo & $3,5 \mathrm{~cm}$ & $\begin{array}{l}\text { Infecciones de } \\
\text { repetición, } \\
\text { obstrucción, } \\
\text { portadora de } \\
\text { nefrostomía }\end{array}$ \\
\hline
\end{tabular}

El número de sesiones ha oscilado entre 1 y 5 , con 2,6 sesiones de media, el 40\% de las litiasis se han resuelto en 1 sesión y el 60\% en 2 sesiones. El número medio de ondas de choque por sesión ha sido de 3961 ondas y las energías han oscilado entre 0,5 y 1,2 mjul/mm2, siendo más altas en las litiasis localizadas a nivel ureteral.

En el 100\% de los pacientes se produjo fragmentación completa de la litiasis, con eliminación de los fragmentos. En 2 pacientes, el 2 y 5, en las litiasis de mayor tamaño 3 y $3,5 \mathrm{~cm}$ precisaron tratamiento antibiótico prolongado y uronefrex para favorecer la eliminación completa de la litiasis residual. El tiempo de tratamiento osciló entre 2 semanas y 6 meses (Tabla II).

\section{DISCUSIÓN}

La formación de cálculos es una de las complicaciones más frecuentes de la derivación urinaria, favorecida por condiciones metabólicas, 
CARACTERÍSTICAS DE LA LITOTRICIA

\begin{tabular}{|c|c|c|c|c|c|c|}
\hline Caso & $\begin{array}{c}\mathbf{N}^{\circ} \\
\text { sesiones }\end{array}$ & No ondas/sesión & Energia/sesión & Fragmentación & Eliminación & $\begin{array}{c}\text { Meses hasta el } \\
\text { alta }\end{array}$ \\
\hline 1 & 4 & $\begin{array}{l}4500 \\
4500 \\
4000 \\
4000\end{array}$ & $\begin{array}{c}0,8 \text { a } 1,4 \\
\mathrm{mjul} / \mathrm{mm} 2\end{array}$ & Total $<2 \mathrm{~mm}$ & Total & 3 \\
\hline 2 & 1 & 5000 & $0,8 \mathrm{mjul} / \mathrm{mm} 2$ & Total & Total & 2,5 \\
\hline 3 & 2 & $\begin{array}{l}4000 \\
3500\end{array}$ & $1 \mathrm{mjul} / \mathrm{mm} 2$ & Total & Total & 1 \\
\hline 4 & 1 & 3500 & $1,2 \mathrm{mjul} / \mathrm{mm} 2$ & Total & Total & 0,5 \\
\hline 5 & 5 & $\begin{array}{l}4000 \\
4000 \\
3500 \\
3500 \\
3500\end{array}$ & $\begin{array}{c}0,8 \text { a } 1 \\
\text { mjul/mm2 }\end{array}$ & Total & Total & 6 \\
\hline
\end{tabular}

infecciosas y anatómicas, estando presente en más de un $12 \%$ de los $\operatorname{casos}^{7}$.

A la hora de elegir un tratamiento hemos de decantarnos, dentro de los más eficaces, por el que sea menos agresivo, ya que los pacientes con derivación urinaria al tener una cirugía previa muy agresiva son propensos a las complicaciones, además, al haber pasado por una intervención tan importante, son muy reacios a pasar por una nueva operación y prefieren maniobras poco agresivas. La litotricia extracorpórea por ondas de choque reúne las características de eficacia y mínima agresividad, siendo de elección en este tipo de pacientes. El porcentaje de pacientes libres de litiasis en la literatura oscila entre el $25 \%$ de Cass y cols. ${ }^{8}$ y el $75 \%$ de Deliveliotis ${ }^{7}$. En nuestro hospital hemos conseguido el $100 \%$ de pacientes libres de litiasis, comprobado por ecografia y radiografia. Esta alta eficacia se debe sobre todo a ser litiasis de origen infeccioso en su mayoría, las cuales se resuelven bien con litotricia, además de haber empleado mayor número de sesiones que los autores antes citados y haber hecho un seguimiento de más duración y uso de antibióticos y uronefrex ${ }^{\circledR}$, para favorecer la eliminación de las litiasis residuales en el caso de las litiasis infecciosas de gran tamaño. A esto hay que añadir que sólo en un caso existía estenosis en la reimplantación ureteral, lo cual dificulta la eliminación de las litiasis.

Hemos tenido una complicación importante, con la presencia de pielonefritis en un paciente que ha precisado ingreso hospitalario para su tratamiento. Los pacientes con diversión urinaria presentan infecciones de repetición con gran frecuencia, por lo cual hay que ser cautos en el tratamiento de estos enfermos y usar antibióticos como profilaxis y derivaciones tipo nefrostomía en caso de complicaciones infecciosas con obstrucción. En nuestra casuística 3 de los cinco enfermos han precisado nefrostomía. Esta alta frecuencia de infecciones hace que sea muy importante el conseguir que el paciente quede libre de litiasis, ya que los restos de la misma favorecen la infección y la formación de nuevas litiasis, por lo que hemos tenido una alta tasa de retratamientos, con 2,6 sesiones de media por paciente.

\section{CONCLUSIONES}

Los pacientes con cistectomía radical y derivación urinaria presentan una predisposición anatómica, metabólica e infecciosa a formar litiasis. Son pacientes que han sido sometidos a una gran variedad de intervenciones y seguimientos, por lo 
que la litotricia puede ser un arma terapéutica importante debida a su baja morbilidad con unos resultados tan satisfactorios en el tratamiento de las litiasis como en los pacientes sin derivación urinaria.

\section{REFERENCIAS}

1. SULLIVAN JW, GRABSTALD H, WHITMORE WF Jr.: Complications of ureteroileal conduict with radical cystectomy. Review of 336 cases. J Urol 1980; 124: 797-801.

2. KOSKO JW, KURSH ED, RESNICK MI.: Metabolic complications of urologic intestinal substitutes. Urol Clin N Am 1986; 13: 193-200.

3. DRETLER SP. The pathogenesis of urinary tract calculi occurring after ileal conduct diversion I: clinical study II: conduit study III: prevention. J Urol 1973; 109: 204-209.

4. TURK TM, KOLESKI FC, ALBALA DM.: Incidence of urolithiasis in cystectomy patients after intestinal conduit or continent urinary diversion. World J Urol 1999; 17: 305-307.
5. COHEN TD, STREEM SB, LAMMERT G.: Long term incidence and risks for recurrent stones following contemporary management of upper tract calculi in patients with urinary diversion. J Urol 1996; 155: 69-70.

6. COHEN TD, STREEM SB, LAMMERT GK.: Selective minimally invasive management of calculi in patient with urinary diversions. J Urol 1994; 152: 10911094.

7. DELIVELIOTIS C, VARKARAKIS J, ARGIROPOULOS $\mathrm{V}$ et al. Shockwave lithotripsy for urinary stones in patients with urinary diversion after radical cystectomy. J Endourology 2002; 16: 717-720.

8. CASS AS, ALIABADI H.: Extracorporeal shock wave lithotripsy and endoscopic management or renal calculi with urinary diversion. J Urology 1992; 148: 1123-1125.

Dr. D. A. Vega Vega

Gran Vỉa de San Marcos, 42 - 6ํㅡ B

24002 León

(Trabajo recibido el 20 mayo de 2003) 